\title{
Deconvolution of ultrasonic echoes using Bernoulli-Gaussian processes for composite materials inspection
}

\author{
A. Benammar ${ }^{1, a}$, R. Drai ${ }^{1}$, A. Kechida ${ }^{1}$ and A. Guessoum ${ }^{2}$ \\ 1 Image and signal processing laboratory, Welding and NDT Centre, route de Dely-Ibrahim, BP 64, Chéraga, \\ 16300 Alger, Algeria \\ ${ }^{2}$ University of Blida, Algeria
}

Received 05 December 2007, accepted 29 March 2008

\begin{abstract}
In this work, we present an approach of deconvolution ill-posed problems of superimposed signals in time. A priori information must be taken into account to solve this problem. The a priori information translates the physical properties of the ultrasonic signals. The defect impulse response is modelled as a Bernoulli-Gaussian sequence. Deconvolution becomes the detection problem of the optimal Bernoulli sequence and estimation of the associated complex amplitudes. A simulation study on defect detection was realised, and results were validated experimentally on Carbon fiber-reinforced polymer multilayered composite materials (CFRP) with and without delamination defects taken from aircraft.
\end{abstract}

Key words: Ultrasonic NDE; composite materials; blind deconvolution; processes BG.

\section{Introduction}

Due to their great potential in weight saving, fiber reinforced laminated composites are becoming increasingly important in applications where low weight, high stiffness, and high strength is required, in particular the aircraft industry. Due to their complex structure, several failure modes occur in laminated composites. Depending on the failure location ply failure and delamination are distinguished. Delamination is caused by out-of-plane shear and normal stresses attributed to e.g. impact loads, free edge effects, ply drop offs, or transverse loading. In this field, ultrasonic testing associated to signal processing must be developed in order to respond to defect detection and evaluation [1-3].

In this paper, we present an approach of deconvolution problem from the estimation of superimposed signals using Bernoulli-Gaussian process $[4,5]$. Section 2 describes the echo detection schemes. In Section 3, we present the deconvolution of backscattered echoes starting with the model of signal and reflectivity estimation. Then we give the results on the synthetic ultrasonic traces. This stage permits to see the robustness of the developed algorithm. In Section 5, we present the experimental results with a carbon fibre reinforced polymer (CFRP) block have been carried out in order to assess the good performance of the proposed approach. Flaw echoes are detected even when they correspond to small defects close to the surface.

\footnotetext{
${ }^{a}$ Corresponding author: Abs_benammar@yahoo.fr
}

\section{Echo detection schemes}

Typically, delaminations in thin composite laminates are detectable by an immersion transducer operating in pulseecho mode. Figure 1a shows a typical ultrasonic system.

The used sample is Carbon fiber-reinforced polymer multi-layered composite materials (CFRP) achieved with two delamination defects located at front surface and back wall.

Multiple signals are reflected from the surfaces of the specimen as well as from delaminations, as shown in Figure $1 \mathrm{~b}$. Typical waveforms recorded by an oscilloscope are shown in Figure 2, where Figure 2a is an A-scan in an undamaged zone and Figures $2 \mathrm{~b}$ and $2 \mathrm{c}$ are the waveform obtained from impact damaged regions of a composite laminate. Figures $2 \mathrm{~b}$ and $2 \mathrm{c}$ show echoes between delamination and respectively, back wall echo and front surface echo. $\tau_{F S}$ represents the time locations of the ultrasonic front surface echo; $\tau_{B}$ represents the time location of the ultrasonic first back wall echo of the sample; $\left(\tau_{B}-\tau_{F S}\right)$ represents the pulse-echo time delay between a front surface and back wall echoes; $\tau_{d}$ represents the defect echo location of the delamination zone. The delamination depth in the material can be given using the equation: $d=\left[V_{\text {sample }}\left(\tau_{d}-\tau_{F S}\right)\right] / 2$ where, $V_{\text {sample }}$ is the velocity of ultrasound in the material.

\section{Deconvolution Bernoulli-Gaussian}

Deconvolution is a well established procedure to remove or reduce the effects of the measurement system for 
(a)

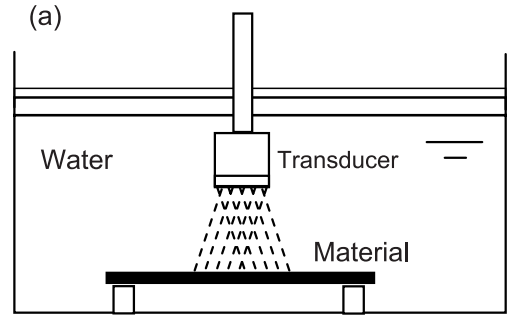

(b)

\section{Reflected waves}

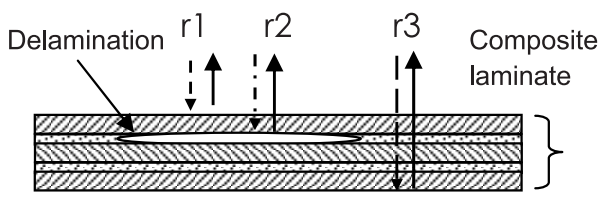

Fig. 1. (a) Experimental setup for immersion pulse-echo testing. (b) Schematic of reflected echoes.
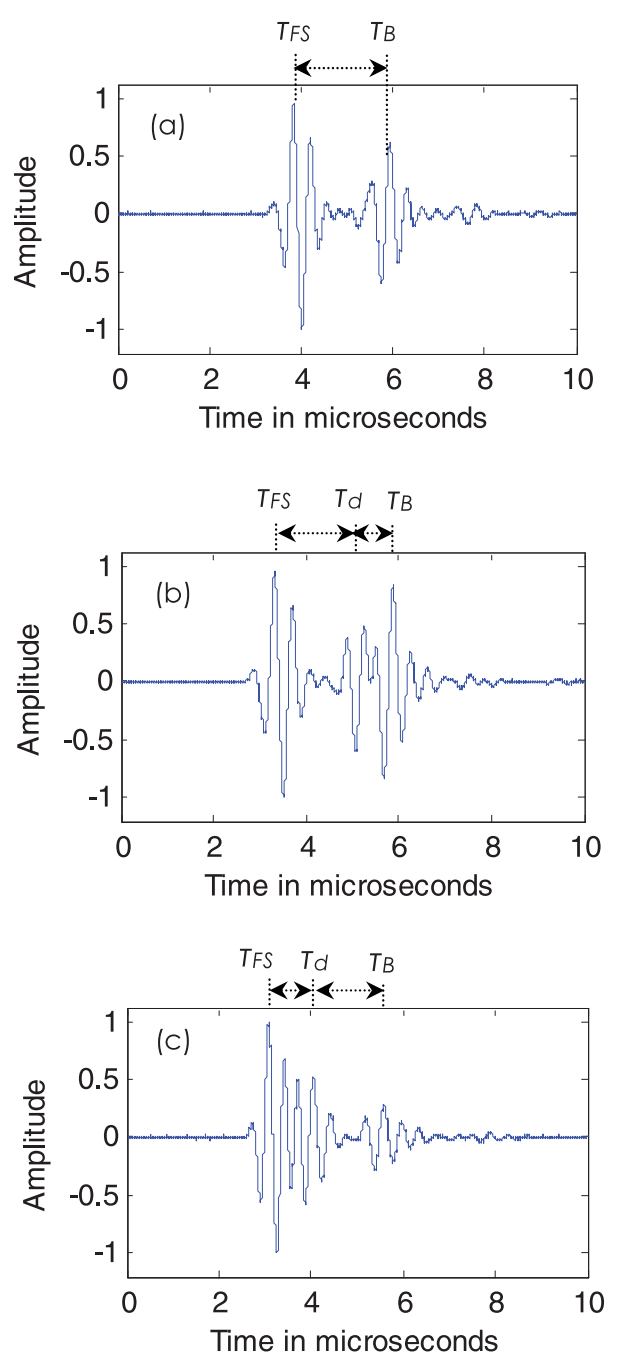

Fig. 2. (a) Waveform in undamaged region, (b, c) Waveforms in damaged region, (b) Closely echoes between delamination and back wall echo, (c) Closely echoes between delamination and front surface echo.

improved resolution. The development of deconvolution has been closely associated with progress in estimation theory, signal processing techniques, and improvements in computational power. A block diagram of the process is given by Figure 3 [6-8].

For a linear time-invariant system, the received signal $x(t)$ is given by:

$$
x(t)=r(t) * h(t)+n(t) .
$$

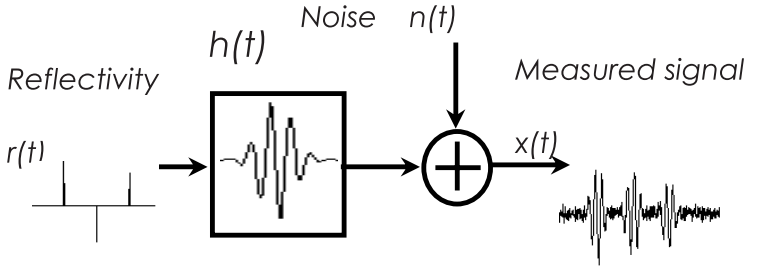

Fig. 3. System model for deconvolution.

Deconvolution provides an estimate of $r(t)$ that satisfies a well-defined optimality criterion based on die measurements of $x(t)$ and knowledge of $h(t)$. Thus, deconvolution requires a priori information of the system, which presents difficulty in its implementation.

The adopted approach, in this work, is the statistical approach introduced initially by Kormylo and Mendel. Each sample of the reflectivity function is supposed to be the realization of a Bernoulli-Gaussian process (BG) $[4,5]$.

The reflectivity sequence is composed a prior of some impulses very localised. The most intuitive formulation of the Bernoulli-Gaussian process is expressed in the shape of a product between two random variables (R.V):

$$
r(k)=t(k) a(k)
$$

where $t(k)$ is the random variable which controls the temporal reflectors position.

This R.V is governed by a law of Bernoulli of $\lambda$ parameter:

$$
\left\{\begin{array}{l}
p(t(k)=1)=\lambda \\
p(t(k)=0)=1-\lambda
\end{array}\right.
$$

and $a(k)$ is a centered Gaussian R.V which controls the amplitudes associated with each reflectors. A low value of $\lambda$ permits to generate a not very dense sequence of impulses. The definition of process BG in this shape of product was introduced by Kormylo and Mendel [4,5]. Thereafter, other researchers such as Goussard et al. adopted a slightly different model being able to be interpreted like a mixture of two centered gaussian. Champagnat recently proposed an extension of model BG permiting to treat such signals [9]. The extension suggested is based on a traditional matrix representation of the convolution equation (4):

$$
x=H r+n .
$$


But with definitions of $H$ and $r$ slightly modified:

$$
H=\left[\begin{array}{ll}
H_{1} & H_{2}
\end{array}\right] \text { et } r=\left[\begin{array}{l}
r_{r e} \\
r_{i m}
\end{array}\right]
$$

where $H_{1}$ and $H_{2}$ are two matrixs whose columns are obtained respectively by shift of $h(t)$ and $T H[h(t)]$.

The required reflectivity can be expressed like the real part and the imaginary part of a function of complexes reflectivity. Each component of this reflectivity is modelled by a Bernoulli-Gaussian process. The new process, named "Double Bernoulli Gaussian" (DBG), is a white process.

The noise $n$ is supposed to be a white gaussian noise centered with variance $\sigma_{n}$. This choice is well adapted to model the electronic noise and the noise of quantification but is not adapted to express the structure noise. The deterministic parameters of which the densities of a prior probability, $\left(\lambda, \sigma_{r}^{2}, \sigma_{n}^{2}\right)$ are called hyperparameters of the problem, and are presupposed known. Indeed, in the case of ultrasonic signals, it is generally possible to estimate their values rather precisely. The variance of the noise $\sigma_{n}^{2}$ is estimated on part of the signal comprising only noise, the variance of signal $\sigma_{r}^{2}$ is a value lower or equal to one, the parameter $\lambda$ is fixed according to the approximate number of reflectors composing the reflectivity sequence and the total number of points of the reflectivity sequence. Knowing the impulse response of the system and the value of the hyperparameters, the goal is to restore the complex reflectivity function.

The estimation of the parameters governing the distribution of random variables is classically obtained by maximizing a probability function. This approach rests on a simple intuitive idea. According to the parameters, the achievements of the random variables take various values. Thus for a given realization, it is more probable than this realization was obtained with a certain set of parameters rather than another. The estimation of the parameters within the sense of the maximum likelihood (ML) consists in determining the value of the parameters which allots to the data observed the greatest probability taking into account description statistics and of the model selected.

The probability of parameters $\theta$ is by definition proportional to the probability density of observations $x$ knowing the true value of the parameters:

$$
V(\theta) \propto p(x \mid \theta) .
$$

The estimate of the sequence of Bernoulli is obtained by maximization of marginal probability a posteriori:

$$
\hat{t} \Delta \underset{t}{\arg \max }[p(t \mid x)]
$$

Marginal probability a posteriori is expressed, by observing the rule of Bayes, in the form equation (8):

$$
V_{M P}(t) \propto p(t \mid x) \propto p(x \mid t) p(t) .
$$

The expression of each of the two terms component $V_{M P}(t)$ is easy to determine. For a sequence $t$ given, $x$ is
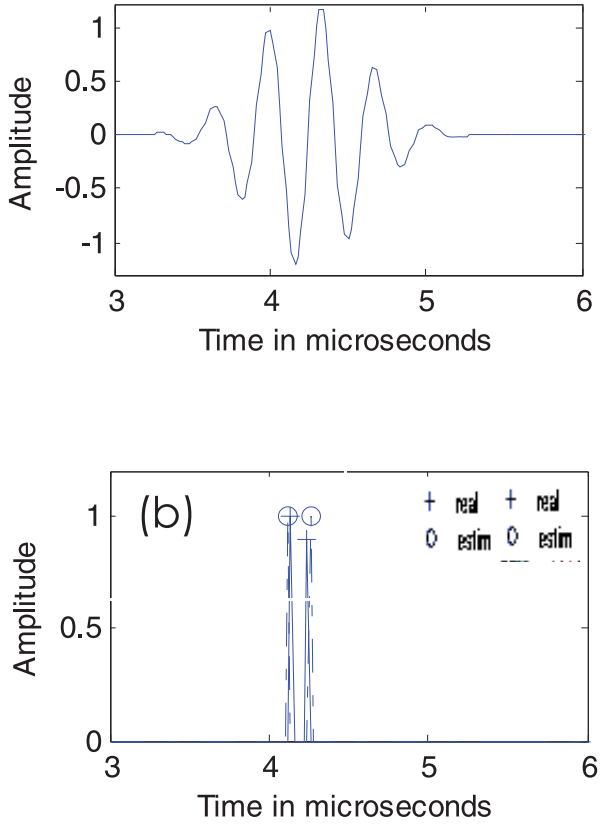

Fig. 4. (a) Closely echoes with noise free. (b) Deconvolution echoes for (a).

a centered Gaussian random variable (because it is the sum of two R.V Gaussian centered) of which the probability density is expressed in the form equation (9):

$$
p(x \mid t)=\frac{1}{\sqrt{(2 \pi)^{K}|N|}} \exp \left(-\frac{x^{t} N^{-1} x}{2}\right)
$$

where, $K$ represents the total number of points of the signal, $N$ is the covariance matrix.

According to the definition of $x$, the covariance matrix is equal to:

$$
N=\sigma_{r}^{2} H \Pi H^{t}+\sigma_{n}^{2} I
$$

where $\Pi$ is proportional to the covariance matrix of $r$ knowing $t$ which, in case of a process DBG, is written:

$$
\Pi=\left[\begin{array}{ll}
T & 0 \\
0 & T
\end{array}\right] \text { with } T \Delta \operatorname{diag}\{t(k)\} .
$$

The research of the relative maximum of the probability function a posteriori is generally realized thanks to the SMLR (single most-likely replacement), $a$ procedure of iterative exploration suggested by Kormylo and Mendel $[4,5]$. From an initial sequence $t_{0}$, all the achievements of $t$ differing from $t_{0}$ of only one of its components are tested.

\section{Synthetic ultrasonic traces}

In order to apprehend the limits of this method, we simulated a signal with very closer echoes. The signal of Figure 4 is obtained by convolution the response impulse of a plane reflector with a transducer of $2.25 \mathrm{MHz}$ frequency and a reflectivity sequence of two reflectors located at the instants $t_{1}=4.12 \mu \mathrm{s}$ and $t_{2}=4.26 \mu \mathrm{s}$. 

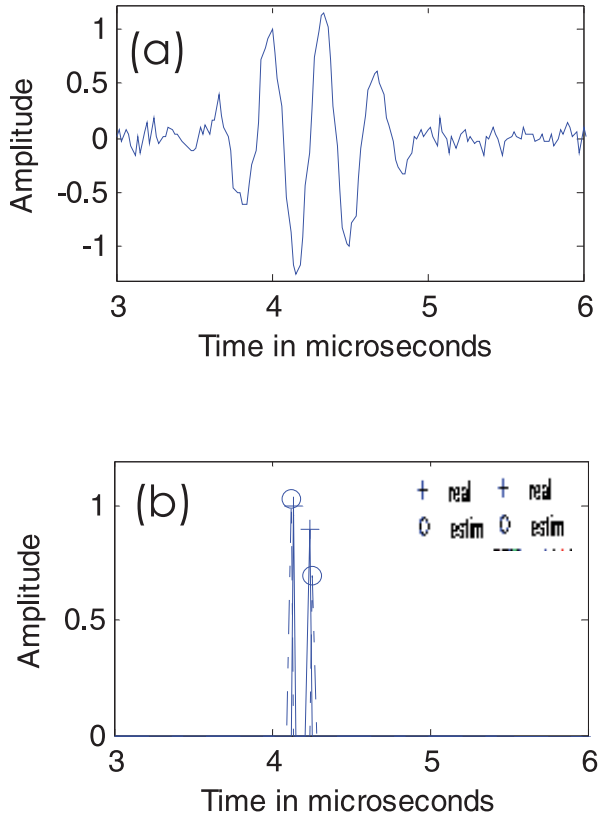

Fig. 5. (a) Two closely spaced overlapping echoes with a SNR of $8 \mathrm{~dB}$. (b) Deconvolution echoes for (a).

The algorithm could detect the two closers echoes with $\Delta \tau=0.14 \mu \mathrm{s}$.

\section{Experimental results}

The experimental data examined in this work was obtained using a $2.25 \mathrm{MHz}$ centre frequency transducer. As sample, a carbon fibre reinforced polymer (CFRP) of $2.7 \mathrm{~mm}$ thickness is used. It provided by the aircraft manufacturer company. It achieved as follow: the unidirectional layers were sticked with epoxy, one layer on the other altering the orientation from $\left(0^{\circ}, 45^{\circ}, 0^{\circ}\right)$. This sample is made of 6 layers of $0.45 \mathrm{~mm}$ thickness each, shared into 3 parts as follow:

- 1st part with no defect;

- 2nd part with a delamination defect past at the end of the 1st layer;

- 3rd part with a delamination defect before the end of the last layer.

Longitudinal waves were used, the ultrasound velocity in this material is $V_{\text {sample }}=2830 \mathrm{~m} / \mathrm{s}$.

Figure 6 shows a signal in the undamaged zone, the arrival time of front surface echo $\tau_{F S}=3.97 \mu$ s and arrival time of back wall echo $\tau_{B}=5.9 \mu \mathrm{s}$. Then thickness of material is $d=\left[V_{\text {sample }}\left(\tau_{b}-\tau_{F S}\right)\right] / 2=2.73 \mathrm{~mm}$.

Figure 7 shows a delamination defect close to the back wall, after application of the deconvolution we have a delamination defect in a depth of $\tau_{d}=1.76 \mu \mathrm{s}$ which is equal to a depth of $2.49 \mathrm{~mm}$. The arrival time of front surface echo $\tau_{F S}=3.45 \mu$ s and arrival time of back wall echo $\tau_{B}=5.69 \mu \mathrm{s}$. The thickness of material in this point is $3.16 \mathrm{~mm}$ this means that we have a delamination of $0.43 \mathrm{~mm}$.

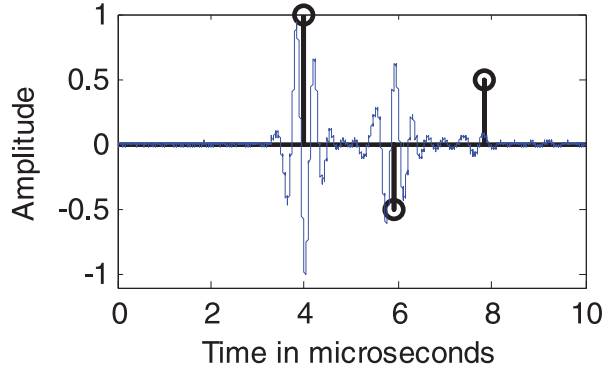

Fig. 6. Waveform in undamaged region and deconvolution result.

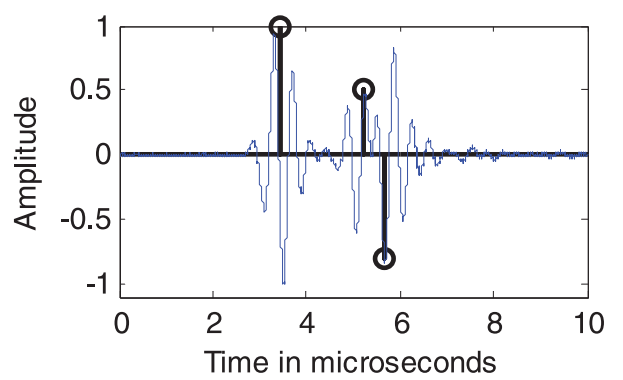

Fig. 7. Waveform in damaged region and deconvolution result (closely echoes in back wall region).

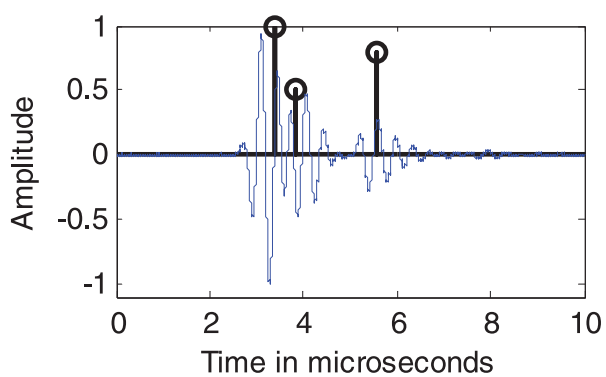

Fig. 8. Waveform in damaged region and deconvolution result (closely echoes in front face region).

Figure 8 shows a delamination defect close to the front surface, after application of the deconvolution we have a delamination defect in a depth of $\tau_{d}=0.45 \mu \mathrm{s}$ which is equal to a depth of $0.63 \mathrm{~mm}$. The arrival time of front surface echo $\tau_{F S}=3.39 \mu$ s and arrival time of back wall echo $\tau_{B}=5.58 \mu \mathrm{s}$. The thickness of material in this point is $3.09 \mathrm{~mm}$ this means that we have a delamination of $0.36 \mathrm{~mm}$.

\section{Conclusion}

In this study, we have developed an approach of deconvolution ill-posed problems of superimposed signals in time. The defect impulse response is modelled as a BernoulliGaussian sequence. The experimental part which validated these results enabled us to determine with precision the position of different delamination defects. We could detect the delamination defect of closer echoes to the back wall at around $0.43 \mathrm{~mm}$ and we detected a 
delamination defect of closer echoes to the front face at around $0.63 \mathrm{~mm}$.

According to the obtained results, we can note that the used and implemented algorithm, could emphasize echoes and consequently the NDT results diagnosis shall be righter.

\section{References}

1. S. Sihn, R.Y. Kim, K. Kawabe, S.W. Tsai. Composites Science and Technology 67, 996 (2007)

2. R. Drai, A. Benammar, Ultrasonic signal processing in the detection of defect in composite, Proceedings of 9th European Conference on NDT (Berlin, Germany, 2006)

3. V.V. Gerasimov, V.S. Khandetsky, S.N. Gnoevoy, Research of probability characteristics in defect detection of compos- ite materials using wavelet transform, Proceedings of 8th International conference of the Slovenian Society for NDT (Portoroz, Slovenia, 2005), pp. 209-215

4. J.J. Kormylo, J.M. Mendel. IEEE Trans. Inf. Theory 28, $482(1982)$

5. J.M. Mendel, Maximum-likelihood deconvolution. A journey into modelbased signal processing, Springer-Verlag, ISBN 0-387-97208-0 (1990)

6. J. Xin, N.M. Bilgutay, Ultrasonic range resolution eniiancement using L1 norm deconvolution, IEEE Trans. Ultrasonics Symposium, 711-714 (1993)

7. J. Idier, Y. Goussard. IEEE Trans. Geosci. Remote Sens. 28, 975 (1990)

8. S.F. Yau, Y. Bresler. IEEE Trans. Signal Process. 41, 804 (1993)

9. F. Champagnat, J. Idier, G. Demoment. Proc. Int. Conf. ASSP 93 3, 452 (1993) 